\title{
Peramine and indole-diterpene biosynthetic capability of epichloë endophytes as predicted by perA and Itm gene analysis
}

\author{
B. SCOTT ${ }^{1}$, C.A. YOUNG ${ }^{1}$, B.A. TAPPER ${ }^{2}$, R.E. WRENN ${ }^{1}$, S.J. FOSTER ${ }^{1}$, C.D. MOON ${ }^{3}$, C.L. SCHARDL SH $^{3}$ \\ ${ }^{I}$ Centre for Functional Genomics, Institute of Molecular BioSciences, Massey University, \\ Private Bag 11 222, Palmerston North, New Zealand \\ ${ }^{2}$ AgResearch, Grasslands Research Centre, Tennent Drive, Private Bag 11 008, Palmerston North New Zealand \\ ${ }^{3}$ University of Kentucky, Department of Plant Pathology, 201 PSB, 1405 Veterans Dr., Lexington, KY 40546-0312, U.S.A.
}

d.b.scott@massey.ac.nz

Genes for the synthesis of the bioprotective metabolites, peramine and indole-diterpenes, were recently cloned from Neotyphodium lolii and Epichloë festucae (Tanaka et al. 2005; Young et al. 2005; Young et al. 2006). Peramine is synthesised by a single multifunctional non-ribosomal peptide synthetase, PerA, comprised of two adenylation and two thiolation domains, and single condensation, methylation and reductase domains (Tanaka et al. 2005). By contrast the indole-diterpenes are a structurally diverse group of metabolites that includes lolitrem B, the most abundant indole-diterpene found in the $N$. lolii-perennial ryegrass association. At least 10 genes are required for lolitrem $B$ biosynthesis $(\mathrm{ltm})$. These form a complex gene cluster composed of three mini-clusters interspersed with AT-rich retrotransposon relic sequence (Young et al. 2006). The aim of this study was to determine the perA and ltm gene composition and distribution among epichloë endophytes, including representative strains of the ten sexual Epichloë species and a taxonomically diverse set of strains from the asexual Neotyphodium species, and on the basis of this analysis predict the symbiotum chemotype.

Using primers designed to conserved regions of the A1 and A2 domains of PerA, PCR products were amplified from all strains of the 21 Epichloë and Neotyphodium species screened, indicating that perA sequences are widely distributed in these genera. However, not all strains have the ability to synthesise peramine in grass symbiota. DNA sequence analysis of the perA 3' sequence from $N$. lolii strain Lp14 identified several mutations in the 3' end of the gene including a premature stop codon at the beginning of the $\mathrm{C}$ domain. Several other strains were highly polymorphic in the perA 3' region indicating that grass symbiota containing these strains will also have a peramine negative phenotype.
By contrast ltm genes showed very limited distribution within the 21 Epichloë and Neotyphodium species screened, principally being confined to E. festucae and their asexual Neotyphodium derivatives. Some strains had the full $\mathrm{ltm}$ gene complement and were able to synthesise a range of lolitrems in grass symbiota. A sub-set of the E. festucae strains examined lacked all 10 genes, explaining the complete absence of indole-diterpenes in symbiota containing these strains. Other strains contained sequences to the eight genes that comprise clusters one and two and synthesised less complex indole-diterpenes in planta. Remnants of ltm genes were detected in some Epichloë species, indicating that this biosynthetic capability is ancestral, having been lost in many lineages.

\section{REFERENCES}

Tanaka, A.; Tapper, B.A.; Popay, A.; Parker, E.J.; Scott, B. 2005. A symbiosis expressed non-ribosomal peptide synthetase from a mutualistic fungal endophyte of perennial ryegrass confers protection to the symbiotum from insect herbivory. Molecular Microbiology 57: 1036-1050.

Young, C.A.; Bryant, M.K.; Christensen, M.J.; Tapper, B.A.; Bryan, G.T.; Scott, B. 2005. Molecular cloning and genetic analysis of a symbiosis-expressed gene cluster for lolitrem biosynthesis from a mutualistic endophyte of perennial ryegrass. Molecular Genetics and Genomics 274: 13-29.

Young, C.A.; Felitti, S.; Shields, K.; Spangenberg, G.; Johnson, R.D.; Bryan, G.T.; Saikia, S.; Scott, B. 2006. A complex gene cluster for indole-diterpene biosynthesis in the grass endophyte Neotyphodium lolii. Fungal Genetics and Biology 43: 679-693. 